\title{
Flattening Process of Polymer Chains Irreversibly Adsorbed on a Solid
}

Mani Sen ${ }^{1}$, Naisheng Jiang ${ }^{1}$, Justin Cheung ${ }^{2}$, Maya K. Endoh ${ }^{1}$, Tadanori Koga ${ }^{*}{ }^{, 1,2,3}$, Daisuke Kawaguchi $^{4}$, Keiji Tanaka ${ }^{*}, 5$

${ }^{1}$ Department of Materials Science and Engineering, Stony Brook University, Stony Brook, NY $11794-2275$

${ }^{2}$ Chemical and Molecular Engineering Program, Stony Brook University, Stony Brook, NY $11794-2275$

${ }^{3}$ Department of Chemistry, Stony Brook University, Stony Brook, NY 11794-3400

${ }^{4}$ Education Center for Global Leaders in Molecular Systems for Devices, Kyushu University, Fukuoka 819-0395, Japan

${ }^{5}$ Department of Applied Chemistry, Faculty of Engineering, Kyushu University, Fukuoka 8190395, Japan 


\section{Experimental Section}

Ellipsometry. The thicknesses of the PS spin coating and dip coating films were measured using an AutoEL-II ellipsometer (Rudolph Research) at room temperature. The wavelength of the laser used in the measurements was $633 \mathrm{~nm}$ and a literature value of 1.589 as a refractive index for $\mathrm{PS}^{1}$ was used for the experiments.

X-ray Reflectivity (XR). XR experiments were performed under ambient conditions at the $\mathrm{X} 10 \mathrm{~B}$ and X20A beamlines of the National Synchrotron Light Source, Brookhaven National Laboratory, and the G2 beamline of Cornell High Energy Synchrotron Source (CHESS). The specular reflectivity was measured as a function of the scattering vector in the direction perpendicular to the surface, $q_{\mathrm{z}}=4 \pi \sin \theta / \lambda$, where $\theta$ is the incident angle and $\lambda$ is the $\mathrm{x}$-ray wavelength $(\lambda=0.087 \mathrm{~nm}$ at $\mathrm{X} 10 \mathrm{~B}, \lambda=0.118 \mathrm{~nm}$ at $\mathrm{X} 20 \mathrm{~A}$, and $\lambda=0.110 \mathrm{~nm}$ at $\mathrm{G} 2$, which are equivalent to the X-ray energies of $14.2 \mathrm{keV}, 10.5 \mathrm{keV}$, and $11.25 \mathrm{keV}$, respectively). The XR data was fit using a standard multilayer fitting routine for a dispersion value ( $\delta$ in the X-ray refractive index) in conjunction with a Fourier transformation (FT) method, a powerful tool to obtain detailed structures for low X-ray contrast polymer multilayers ${ }^{2}$.

Atomic Force Microscopy (AFM) measurements. Surface morphologies of the interfacial sublayers and flattened layers were studied using atomic force microscopy (AFM) (Veeco Multimode V) at The Center for Functional Nanomaterials (CFN) at BNL. Standard tapping mode experiments were conducted in air using cantilevers with spring constants of about 40 $\mathrm{N} / \mathrm{m}$. The scan rate was 0.5 to $1.0 \mathrm{~Hz}$ with a scanning density of 512 lines per frame. 


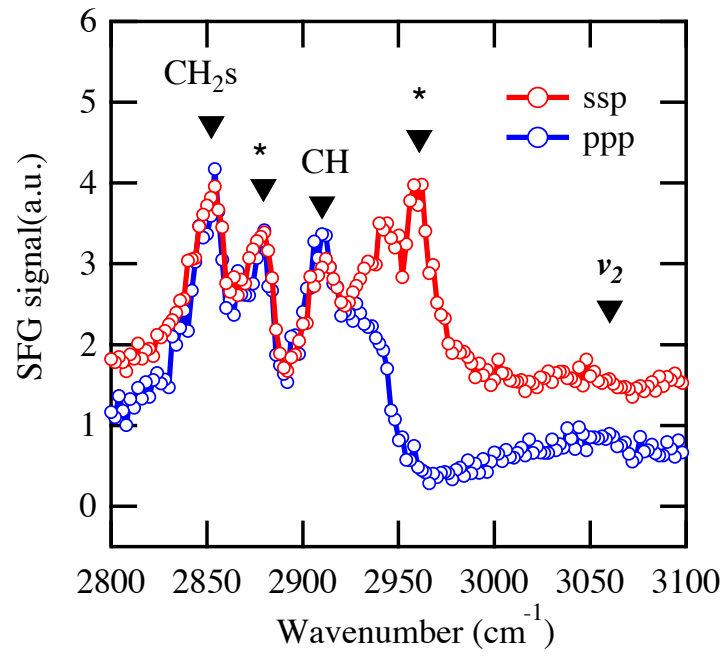

Fig. S1. SFG spectra from the ssp and $p p p$ polarization combinations for the flattened layer derived from the dip-coating film annealed at $150{ }^{\circ} \mathrm{C}$ for $144 \mathrm{~h}$. The assignments of the peaks are described in the main text.

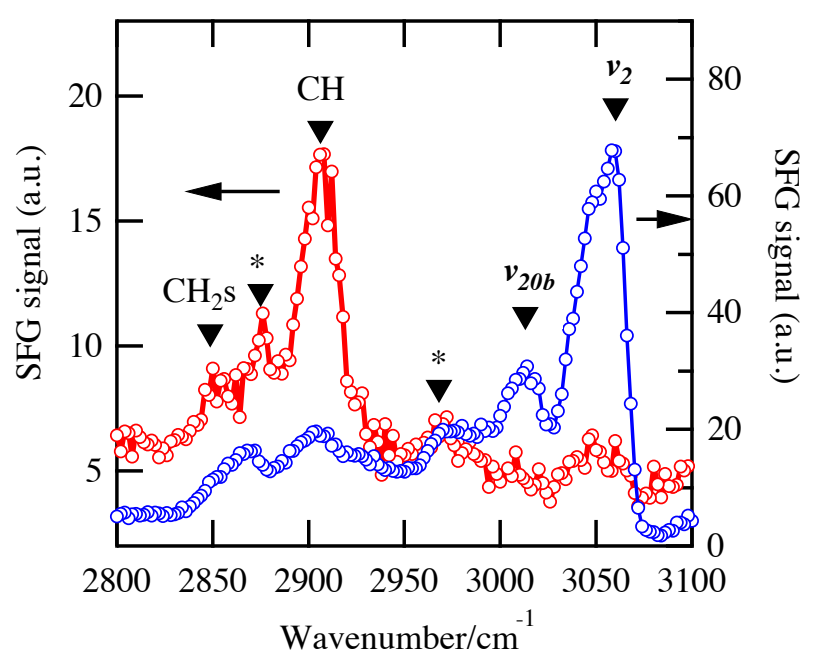

Fig. S2. SFG spectra for the PS spin-cast film annealed for $3 \mathrm{~h}$ (blue) and $144 \mathrm{~h}$ (red) at $150{ }^{\circ} \mathrm{C}$ with the $s s p$ polarization combination. The SFG data for $3 \mathrm{~h}$ annealing is from Ref. 32. Note that the peak around 3020 $\mathrm{cm}^{-1}$ is attributed to the contribution from the $v_{20 \mathrm{~b}}$ vibrational mode of phenyl rings ${ }^{3,4}$. 


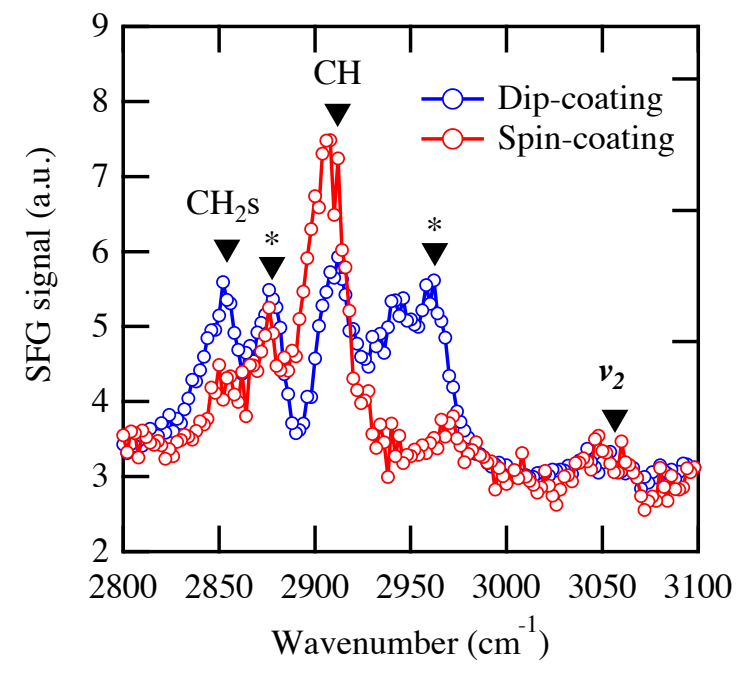

Fig. S3. SFG spectra from the $s s p$ polarization combination for the interfacial sublayers derived from the spin-coating and dip-coating films annealed at 150 ${ }^{\circ} \mathrm{C}$ for $144 \mathrm{~h}$.

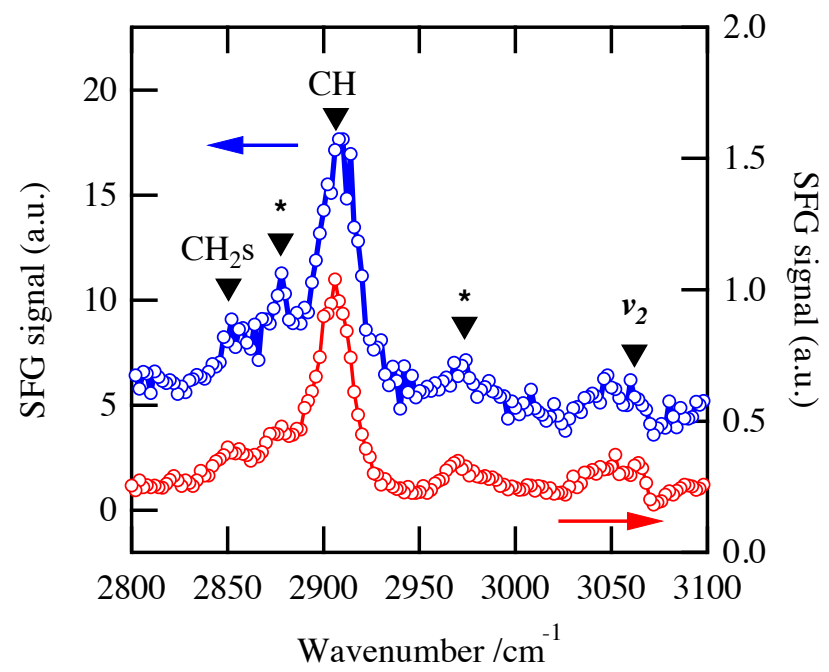

Fig. S4. Comparison of the SFG spectra from the $s s p$ polarization combination for the sandwiched (blue) and uncapped (red) flattened layer derived from the spin-coating film annealed at $150{ }^{\circ} \mathrm{C}$ for $144 \mathrm{~h}$. 


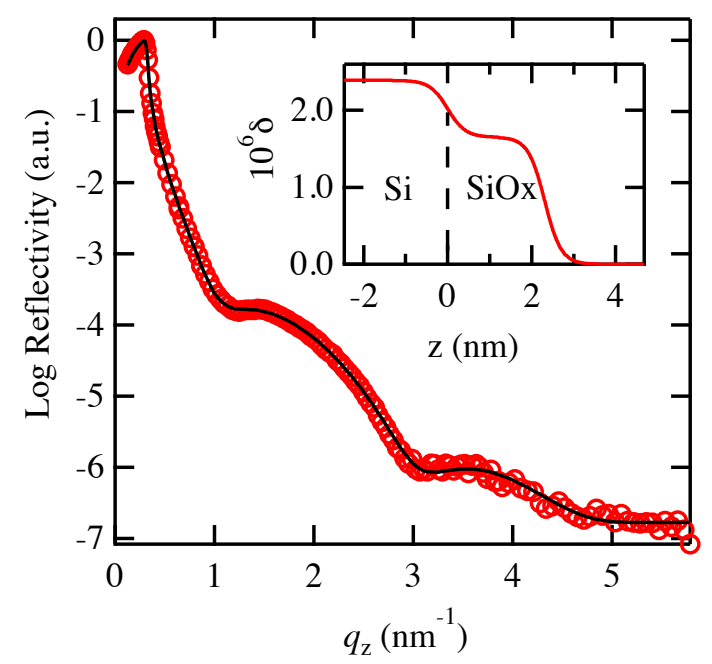

Fig. S5. XR curve of the cleaned $\mathrm{SiO}_{\mathrm{x}} / \mathrm{Si}$ substrate. The black solid line corresponds to the best-fit to the data based on the dispersion $(\delta)$ profile against the distance $(\mathrm{z})$ from the interface between $\mathrm{Si}$ substrate and native oxide layer $\left(\mathrm{SiO}_{\mathrm{x}} / \mathrm{Si}\right.$ interface $)$ as shown in the inset. The dotted line in the inset corresponds to the $\mathrm{SiO}_{\mathrm{x}} / \mathrm{Si}$ interface.

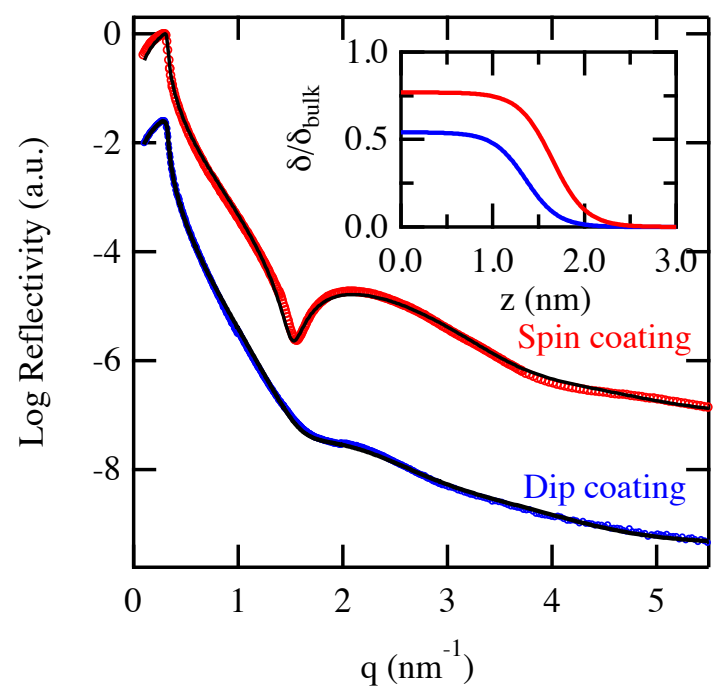

Fig. S6. XR curves of the quasiequilibrium flattened layers. The solid lines correspond to the best-fits to the data based on the normalized dispersion $(\delta)$ profiles (by the bulk $\delta_{\text {bulk }}$ value) against the distance (z) from the SiOx surface shown in the inset: red line, the flattened layer derived from the spin-coated film with the X-ray energy $(E)$ of $10.5 \mathrm{keV}$; blue line, the flattened layer derived from the dip-coated film with $E=11.25 \mathrm{keV}$. The $\delta$ values of the bulk PS are $1.85 \times 10^{-6}$ for $E=11.25 \mathrm{keV}$ and $2.12 \times 10^{-6}$ for $E=10.5 \mathrm{keV}$. 

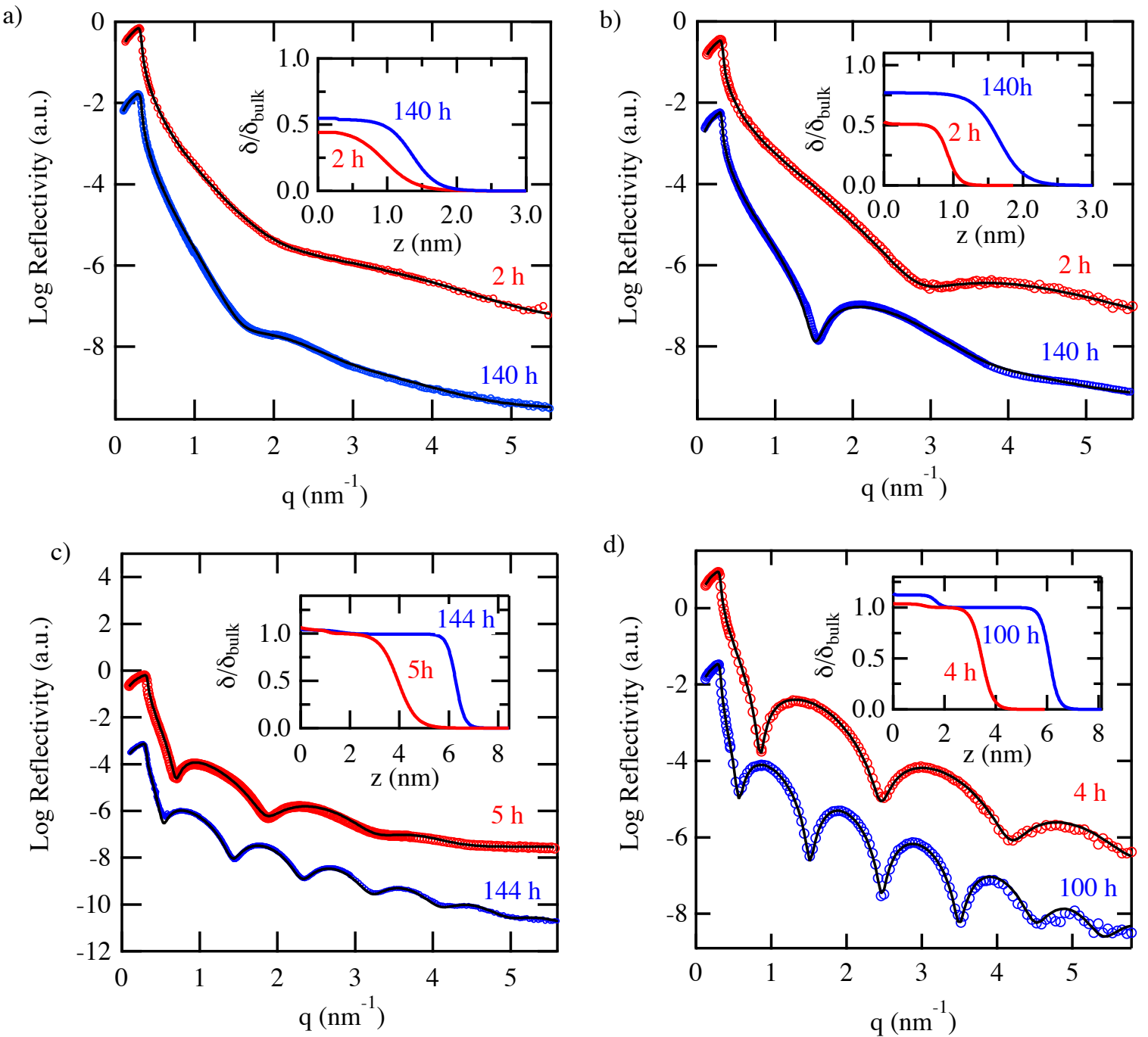

Fig. S7. Representative time dependence of the XR curves of the flattened layers derived from (a) the dip-coating films with $E=14.2 \mathrm{keV}$ (red) and $E=11.25 \mathrm{keV}$ (blue) and (b) spin-coating films with $E=14.2 \mathrm{keV}$ (red) and $E=10.5 \mathrm{keV}$ (blue), and the interfacial sublayers from (c) the dip-coating films with $E=10.5 \mathrm{keV}$ (red) and $E=11.25 \mathrm{keV}$ (blue) and (d) the spin-coating films with $E=14.2 \mathrm{keV}$. The numbers displayed in the figures correspond to the annealing time. The solid lines correspond to the best-fits to the data based on the normalized dispersion $(\delta)$ profiles (by the bulk $\delta_{\text {bulk }}$ value) against the distance (z) from the SiOx surface shown in the inset. The $\delta_{\text {bulk }}$ values of PS are $2.12 \times 10^{-6}$ for $E=10.5 \mathrm{keV}, 1.85 \times 10^{-6}$ for $E=11.25 \mathrm{keV}$, and $1.14 \times 10^{-6}$ for $E=14.2 \mathrm{keV}$. It should be noted that there are two different PS layers with different densities for the interfacial sublayer, as reported previously ${ }^{5,6}$. The choice of the twolayer model was determined by the corresponding Fourier transformation (FT) profile of the XR profile. The details of the XR analysis in conjunction with the FT method for the PS interfacial sublayers have been described elsewhere ${ }^{5,6}$. 

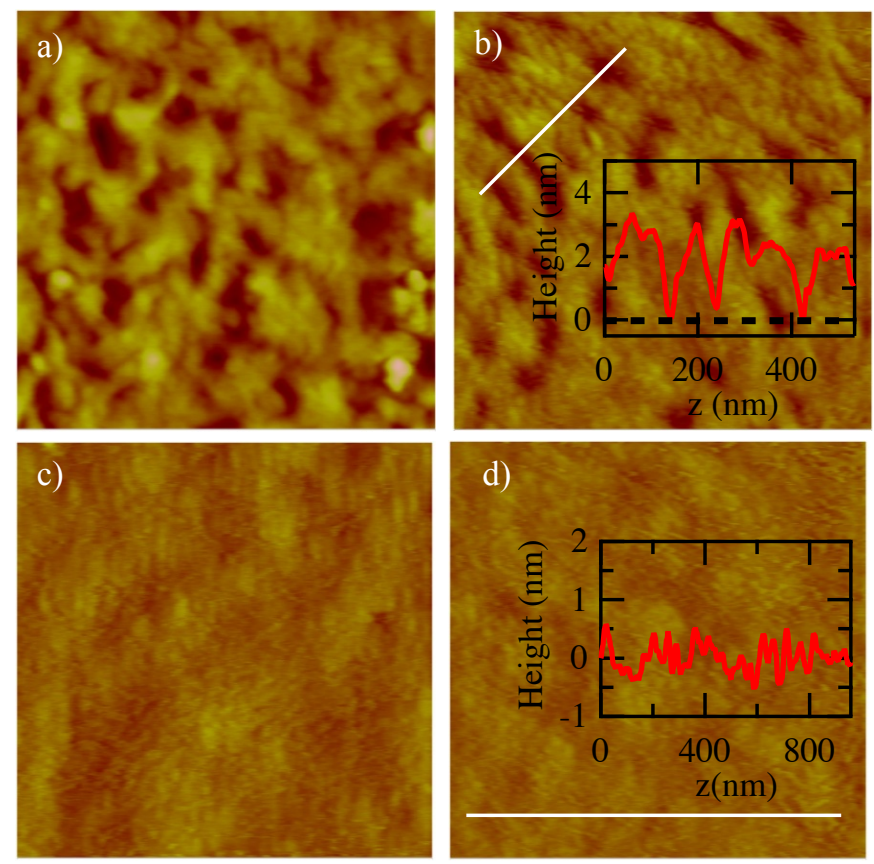

Fig. S8. AFM height images of the PS flattened layer surfaces derived from (a) the dip coating film and (b) the spin-coating film and the interfacial sublayers derived from (c) the dip coating film and (d) the spin-coating film after annealing at $150^{\circ} \mathrm{C}$ for $144 \mathrm{~h}$. The scan sizes and height scales are $1 \mu \mathrm{m}$ $\times 1 \mu \mathrm{m}$ and $0-6 \mathrm{~nm}$ for all the images, respectively. The insets of the images (b) and (d) correspond to the height profiles along the white lines in (b) and (d). The dotted line corresponds to the $\mathrm{SiO}_{\mathrm{x}}$ surface. 
Surface coverage of the flattened layers. In order to estimate the surface coverage $\left(\phi_{\mathrm{p}}\right)$ of the flattened layers, we applied bearing area analysis using the NanoScope Analysis software (version 1.40, Bruker) that we have established previously ${ }^{5}$. A bearing area gives a percentage of the surface above a critical threshold. For this analysis, the AFM height images were used and we set the critical threshold to $0 \mathrm{~nm}$ at the polymer $/ \mathrm{SiO}_{2}$ interface. We independently preformed a scratch test to find the interface. Fig. S9 shows representative bearing analysis results using the AFM images shown in Fig. S8(a) and (b). The average $\phi_{\mathrm{p}}$ value of $48 \%( \pm 5$ $\%$ ) was estimated for the flattened layer derived from the dip-coating based on several AFM images at different spots of the film. The $\phi_{\mathrm{p}}$
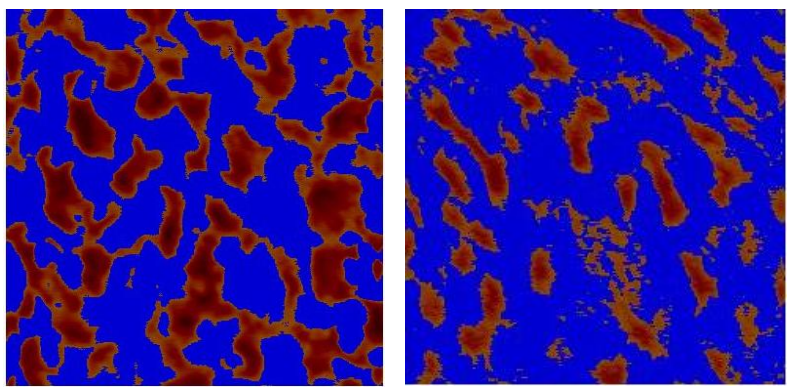

Fig. S9. Bearing area analysis results of the AFM images shown in Fig. S8 (a) (left) and Fig. S8 (b) (right). The areas occupied by the polymer (bearing areas) are colored in blue. value for the flattened layer derived from the spin-coating film was estimated to be $70 \pm 5 \%$.

The observed dispersion value of the flattened layer $\left(\delta_{\text {obs }}\right)$ is given by:

$$
\delta_{o b s}=\delta_{P S} \times \phi_{p}+\delta_{a i r} \times\left(1-\phi_{p}\right)(\mathrm{S} 1)
$$

where $\delta_{\mathrm{ps}}$ and $\delta_{\text {air }}$ are the dispersion values of the PS flattened layer and air, respectively. Eq. (S1) with the given $\delta_{\text {air }}$ (which can be approximated to zero since the value is much smaller than $\left.\delta_{\mathrm{ps}}\right), \phi_{\mathrm{p}}$, and $\delta_{\mathrm{obs}}$ values gives us $\delta_{\mathrm{ps}}=2.1 \times 10^{-6}$ for the flattened layer derived from the dip-coating film and $\delta_{\mathrm{ps}}=2.3 \times 10^{-6}$ for the flattened layer derived from the spin-coating film, respectively. This higher $\delta_{\mathrm{ps}}$ value of the flattened layer derived from the spin-coating film (by about 10\%) is consistent with the previous experimental ${ }^{5-7}$ and simulational findings ${ }^{8,9}$, while the $\delta_{\text {ps }}$ value of the flattened layer derived from the dip-coating film is nearly the same as the bulk $\delta_{\mathrm{ps}}$. At this point, it is not yet conclusive whether the high-density flattened layer is also formed at the polymer-solid interface of the dip-coating film. However, it should be noted that the lateral correlation length of the textures seen in the flattened layer is less than the coherent length of Xray beams at the sample position ( $\sim 1 \mu \mathrm{m}$ in the present case). Therefore, X-rays are sensitive to the textures and would interfere with them, possibly causing a drastic change in the "amplitude" of Kiessig oscillation fringes. This would make the accuracy of the data fitting (for the density and roughness of the flattened layer) somewhat ambiguous, while the thickness can be independently and still accurately determined from the "period" of the oscillation fringes. Hence, it may be difficult to obtain the accurate $\delta$ values for the flattened layers with low surface coverage. Further experiments need to be done to clarify the issue. 
SFG signal from methyl groups. According to a previous report by Müller-Buschbaum and coworkers ${ }^{10}$, it was demonstrated that residual toluene molecules are bound at the substrate interface of PS spin-cast films even after thermal annealing, which may give signals from methyl groups. To rule out the possibility, we independently prepared spin-coating and dip-coating PS $\left(M_{\mathrm{w}}=290,000 \mathrm{~g} / \mathrm{mol}\right)$ films using deuterated toluene as a solvent. The films were then annealed at $150{ }^{\circ} \mathrm{C}$ for $96 \mathrm{~h}$ to evaporate the solvent completely, which was a much longer annealing time compared to that $\left(8 \mathrm{~h}\right.$ at $120^{\circ} \mathrm{C}$ and $\left.160^{\circ} \mathrm{C}\right)$ used in the literature ${ }^{10}$. The SFG experiments clearly detected the signals at $2960 \mathrm{~cm}^{-1}$, which is attributed to the $\mathrm{C}-\mathrm{H}$ anti-stretching vibration of methyl groups (data not shown). Thus, it is reasonable to conclude that the SFG peaks (indicated with asterisks in Fig. 2) are not from any residual solvent molecules. In addition, as reported previously $^{3}$, we characterized a film of PS $\left(M_{\mathrm{w}}=62,000 \mathrm{~g} / \mathrm{mol}\right)$ synthesized by sodium naphthalenide as a bifunctional polymerization initiator so that there were no methyl groups in the polymer chains. The results illuminated no signals from methyl groups in the SFG spectra from the PS thin film. Hence, it is reasonable to conclude that the observation of the signal from methyl groups indicates that a fragment of the initiator, $\mathrm{Sec}-\mathrm{BuLi}$, is segregated at the interface.

Liquid contact angle measurements for $\mathrm{SiOx} / \mathrm{Si}$ and quartz. Static contact angle measurements with two liquids (water and glycerol) were carried out using a CAM 200 optical contact angle meter (KSV instruments, Ltd.) equipped with a video camera. A static contact angle $(\theta)$ of a film surface was determined on the basis of the three-phase contact line with a 2 $\mu \mathrm{L}$ liquid droplet. All the results were obtained by averaging data from at least 5 individual samples and 10 readings per sample at different locations. It was found that the static contact angle of the $\mathrm{SiOx} / \mathrm{Si}$ is $\theta=(18 \pm 1)^{\circ}$ for glycerol, which is in good agreement with that of that the quartz substrate $\left(\theta=(20 \pm 1)^{\circ}\right)$ for glycerol, which is also in good agreement with a previous result $\left(\theta=26^{\circ} \text { for glycerol }\right)^{11}$. 


\section{References}

1. Lu, Y. J.; Xia, H. W.; Zhang, G. Z.; Wu, C. J. Mater. Chem. 2009, 19, 5952-5955.

2. Seeck, O. H.; Kaendler, I. D.; Tolan, M.; Shin, K.; Rafailovich, M. H.; Sokolov, J.; Kolb, R. Appl. Phys. Lett. 2000, 76, 2713-2715.

3. Horinouchi, A.; Yamada, N. L.; Tanaka, K. Langumuir 2014, 30, 6565-6570.

4. $\quad$ Tsuruta, H.; Fujii, Y.; Kai, N.; Kataoka, H.; Ishizone, T.; Doi, M.; Morita, H.; Tanaka, K. Macromolecules 2012, 45, 4643-4649.

5. Jiang, N.; Shang, J.; Di, X.; Endoh, M. K.; Koga, T. Macromolecules 2014, 47, 26822689.

6. Gin, P.; Jiang, N. S.; Liang, C.; Taniguchi, T.; Akgun, B.; Satija, S. K.; Endoh, M. K.; Koga, T. Phys. Rev. Lett. 2012, 109, 265501.

7. Durning, C. J.; O'Shaughness, B.; Sawhney, U.; Nguyen, D.; Majewski, J.; Smith, G. S. Macromolecules 1999, 32, 6772-6781.

8. $\quad$ Kallrot, N.; Dahlqvist, M.; Linse, P. Macromolecules 2009, 42, 3641-3649.

9. Carrillo, J. M. Y.; Cheng, S. W.; Kumar, R.; Goswami, M.; Sokolov, A. P.; Sumpter, B. G. Macromolecules 2015, 48, 4207-4219.

10. Perlich, J.; Körstgens, V.; Metwalli, E.; Schulz, L.; Georgii, R.; Müller-Buschbaum, P. Macromolecules 2009, 42, 337-344.

11. Janczuk, B.; Zdziennicka, A. J. Mater. Sci. 1994, 29, 3559-3564. 